\title{
Details of the mechanism of dissolution of iron-zinc coatings based on the $\delta$-phase in acidic media
}

\section{A.I. Biryukov, ${ }^{1 *}$ O.A. Kozaderov, ${ }^{2}$ (i) R.G. Galin, ${ }^{3}$ D.A. Zakharyevich ${ }^{1}$ and V.E. Zhivulin ${ }^{4,5}$}

${ }^{1}$ Chelyabinsk State University, 129 Br. Kashirinykh str., 454001 Chelyabinsk, Russian Federation

${ }^{2}$ Voronezh State University, 1 Universitetskaya pl., 394018 Voronezh, Russian Federation

${ }^{3}$ Vika Gal Ltd., 7 Molodogvardeytsev str., 454138 Chelyabinsk, Russian Federation

${ }^{4}$ South Ural State University (Research University), 76 Lenin pr., 454080, Chelyabinsk, Russian Federation

${ }^{5}$ South Ural State Humanitarian Pedagogical University, 69 Lenin pr., 454080

Chelyabinsk, Russian Federation

*E-mail: BiryukovAI.csu@yandex.ru

\begin{abstract}
The anodic corrosion behavior of diffusion zinc coatings, the main component of which is the delta phase, was studied in acidic media. Diffusion zinc coatings were obtained by heating in zinc powders, the surface of the particles of which is coated with zinc oxide. Gravimetric measurements, potentiometry, scanning electron microscopy and X-ray structural analysis have been used to study the characteristics of anodic corrosion as well as the evolution of surface morphology. The concentrations of zinc and iron ions were determined in acidic corrosive media by atomic absorption spectroscopy. The concentrations of zinc and iron in the diffusion zinc coatings were determined using X-ray fluorescence spectroscopy. The data obtained were used to calculate the selectivity coefficients of dissolution of elements. It is shown in the work that the dissolution rate nonlinearly depends on the concentration of zinc and the thickness of the coating. It was found that the corrosion of the zinc-rich $\delta$-phase is accompanied mainly by the dissolution of zinc in it. Dezincification is accompanied by surface morphological development and the formation of multiple cracks and pores, which, in turn, initiate galvanic corrosion. Prolonged acidic corrosion of the iron-zinc coating leads to deep dezincification with phase transformation.
\end{abstract}

Keywords: diffusion iron-zinc coatings, selective dissolution, structurally selective corrosion, sherardizing.

Received: November 13, 2020. Published: November 25, 2020

doi: $\underline{10.17675 / 2305-6894-2020-9-4-18}$

\section{Introduction}

Coatings based on intermetallic Fe, $\mathrm{Zn}$-phases are widely used in the protection of steel constructions from corrosion in the oil, automotive, and construction industries. iron-zinc coatings are mainly produced by hot-dip galvanizing (HDG) with further thermal treatment 
which forms galvannealed (GA) coatings [1]. Another less common technique is sherardizing, which involves heating steel in zinc powders with various additives. The process results in the production of so-called diffusion zinc (DZ) coatings [2, 3]. Regardless of the method used to synthesize iron-zinc coatings, they are composed of layers of different $\mathrm{Fe}, \mathrm{Zn}$-phases, where the atomic fraction of zinc increases according to the diagram of the Fe-Zn system as the distance from the surface of the substrate increases [1]. The thickness of the layers of such phases is largely dependent on a coating method. Thus, in case of HDG, it mainly consists of $\eta$-phase layers (>95.0 wt.\%) and the $\zeta$-phase (94.0-95.0 wt.\% of Zn), characterized by a relatively larger thickness [1]. Whereas the structure of GA and DZ coatings might hardly have any $\zeta$ - and $\eta$-phases $[2,3]$, and the base of the coating is the $\delta$ phase with $88.5-93.0 \mathrm{wt} . \%$ of $\mathrm{Zn}$ [1]. It is obvious that the differences in the phase composition of iron-zinc coatings obtained by different methods can have a marked influence on the mechanisms of corrosion processes occurring on their surfaces.

Given the composition of Fe, Zn-coatings obtained by HDG, their corrosion mechanism is usually described similarly to pure zinc $[4,5]$. However, such an approach is no longer applicable to GA and DZ coatings. What is more, according to $[6,7]$ and our own results [8-10], such coatings have greater corrosion resistance than pure zinc or iron-zinc coatings obtained by hot-dip galvanizing. The study of the corrosion behavior of DZ coatings in a chloride-containing solution revealed non-monotonic dependence of the corrosion current on the $\delta$-phase composition on the coating surface with at least $90 \mathrm{wt} . \%$ of $\mathrm{Zn}$ [5]. Taking into account the differences in the phase compositions of coatings, the observed effects can be explained by the predominance of the $\delta$-phase in the composition of GA and DZ coatings. In this regard, there is an urgent task to study the corrosion mechanism of the $\delta$-phase and $\delta$ phase-based coatings.

It is known that the corrosion of alloys in which the metals differ in their electrode potentials can be accompanied by the predominant dissolution of the electronegative component, i.e. it can become selective [11-14]. In [15-23], there were attempts to investigate the corrosion behavior of $\delta$-phase-based coatings in a weak acidic media. It is assumed that the $\delta$-phase is exposed to a selective dissolution of zinc [16, 23], which leads to the appearance of cracks and pores in the coating $[16,23]$. The cracks and defects can permeate the entire coating down to the steel substrate. Thus, both external and internal layers of coating representing different $\mathrm{Fe}, \mathrm{Zn}$-phases can be in contact with the electrolyte. They differ in zinc concentration and have different electrode potentials. The appearance of galvanic coupling between the phases and the occurrence of an uneven three-dimensional dissolution front $[15,17]$ lead to the simultaneous damage of all phases $[15,16,18]$.

In the existing studies, the authors have mainly investigated thin (about $10 \mu \mathrm{m}$ ) HDG coatings which consist of phases with a high zinc content. In this case, the thickness of the $\delta$ phase is small, which nearly eliminates the possibility to study its corrosion mechanism and patterns. In particular, there is no description of the dependency of the corrosion on the chemical composition of the phase. For the same reason, these papers do not explain the mechanism of selective dissolution of zinc from the $\delta$-phase. Moreover, they do not determine 
the nature of the solution products of the $\delta$-phase in acid solutions. Several authors $[15,16,18,19,23,24]$ have observed the formation of black films on the surface of coatings. However, they have different opinions about the nature of these films. For example, in [19], it is shown that black films are only formed in the $\zeta$ - and $\delta$-phases. In $[15,16,24]$, the authors suggest that the film consists of metallic iron or zinc particles and the corrosion products of zinc $\left(\mathrm{Zn}(\mathrm{OH})_{2} \times \mathrm{H}_{2} \mathrm{O}\right)$. In [18], the appearance of the black film is explained by the accumulation of impurities in the coating, such as bismuth. In [23], the formation of a black sponge-like structure is associated with the formation of a solid solution of $\mathrm{Zn}-\mathrm{Fe}$ with a concentration of zinc that is significantly greater than the solubility limit at room temperature.

The goal of the work is to investigate the mechanism of the $\delta$-phase dissolution in diffusion coatings of different thickness and chemical composition and to determine the composition and the structure of the products of the corrosion damage of the $\delta$-phase.

\section{Materials and Methods}

\subsection{Materials}

The objects of our study were diffusion zinc coatings of 5-100 $\mu \mathrm{m}$ in thickness, coated on a carbon steel substrate (steel 45). The surfaces of the steel discs $(\mathrm{d}=24.0-26.0 \mathrm{~mm})$ were polished with fine sandpaper (P1000), their dimensions were measured, and they were weighed with a precision of up to $0.0001 \mathrm{~g}$. Then, the samples were heated to $450^{\circ} \mathrm{C}$ in zinc powder, the particle surfaces of which were coated with nanostructured zinc oxide. The coating technology and the process of the formation of coatings were described in $[25,26]$. After the coatings were formed, the mass of the samples was measured again and the thickness of the coatings was calculated. Their surface was ground with abrasive paper (R2500), polished with a cloth with an aqueous suspension of chromium oxide (III), and then the surface was washed with distilled water and dried. Immediately before the research, the samples were degreased with isopropyl alcohol.

Experiments were carried out in solutions of hydrochloric acid $(0.1 \mathrm{M})$ with the addition of sodium chloride $(0.5 \mathrm{M})$. The solutions were prepared from analytically pure reagents and distilled water.

\subsection{Investigation of the chemical and phase compositions of the surface layers of coatings}

Scanning electron microscopy.

The compositions of the samples were investigated using a Jeol JSM7001F scanning electron microscope equipped with an Oxford INCA X-max 80 energy dispersive X-ray fluorescence spectrometer for elemental analysis.

\section{$X$-ray diffraction.}

$X$-ray diffraction analysis of the initial samples of diffusion zinc coatings, as well as the products of their dissolution in acids was performed on a DRON-3 diffractometer with $\mathrm{Cu} \mathrm{K \alpha}$-radiation, within an angle range of $20^{\circ} \leq 2 \theta \leq 60^{\circ}$ at $1^{\circ} / \mathrm{min}$. 


\subsection{Investigation of electrochemical and corrosion behavior}

\section{Gravimetric measurements}

Samples of the coatings were weighed with a precision of up to $0.0001 \mathrm{~g}$ and were immersed in a solution of $0.1 \mathrm{M} \mathrm{HCl}+0.5 \mathrm{M} \mathrm{NaCl}$. After 5 and 10 minutes of exposure, the samples were removed and washed with running distilled water, dried at room temperature, and weighed with the same accuracy. The difference in weight before and after the exposure was used to calculate the dissolution rate in $\mathrm{mg} / \mathrm{cm}^{2}$ and hours and to evaluate the dissolution depth of coatings in $\mu \mathrm{m}$.

\section{Measurements of open circuit potential (OCP)}

The electrode potential was measured relative to a silver chloride electrode reference electrode in a solution of $0.1 \mathrm{M} \mathrm{HCl}$ for $60 \mathrm{~min}$.

\section{Determination of zinc and iron concentrations in the solutions}

During the dissolution of coatings, samples of the solution were taken at different time intervals and the concentrations of $\mathrm{Zn}^{2+}$ and $\mathrm{Fe}^{2+}$ ions were determined by means of atomic absorption spectroscopy with the help of a KVANT-2a spectrometer with flame atomization.

The selectivity coefficient was calculated, $Z_{\mathrm{Zn}}=\frac{\left(C_{\mathrm{Zn}^{2+}} / C_{\mathrm{Fe}^{2+}}\right)_{\text {solution }}}{\left(C_{\mathrm{Zn}_{\mathrm{n}}} / C_{\mathrm{Fe}}\right)_{\text {alloy }}}$, where $\left(C_{\mathrm{Zn}^{2+}} / C_{\mathrm{Fe}^{2+}}\right)_{\text {solution }}$ is the ratio of the concentrations of zinc and iron ions in the etching solution and $\left(C_{\mathrm{Zn}} / C_{\mathrm{Fe}}\right)_{\text {alloy }}$ is the ratio of the concentrations of $\mathrm{Zn}$ and Fe in the coating. If $Z_{\mathrm{Zn}}>1$, then there is a predominant dissolution of zinc.

\section{Results and Discussion}

\subsection{Physical and chemical characteristics of samples}

Figure 1 shows the dependence of zinc concentration at the surface of the coating on its thickness. It can be seen that as the coating thickness increases from 5 to $40 \mu \mathrm{m}$, the $\mathrm{Zn}$ content therein increases, however, with a further thickening of the coating, the zinc concentration in the coating stabilizes at $92.0-93.0 \mathrm{wt} . \%$.

In the XRD patterns of the coatings, regardless of their thickness, in the angular region of $2 \theta=40-44^{\circ}$ there are peaks corresponding to the iron-zinc phases. What is more, the predominant peaks were the $\delta$-phase peaks $\left(\mathrm{FeZn}_{7-10}\right)$. There were no reflections of pure zinc or peaks of the $\zeta$-phase $\left(\mathrm{FeZn}_{13}\right)$ or zinc oxide on the surfaces of the coatings. Thus, it can be concluded that the surface layers of coatings with a thickness of 20 to $100 \mu \mathrm{m}$, wherein the concentration of zinc is within the interval of 88.0-93.0 wt.\%, only consist of the $\delta$-phase, which agrees with previous works $[9,26]$. 


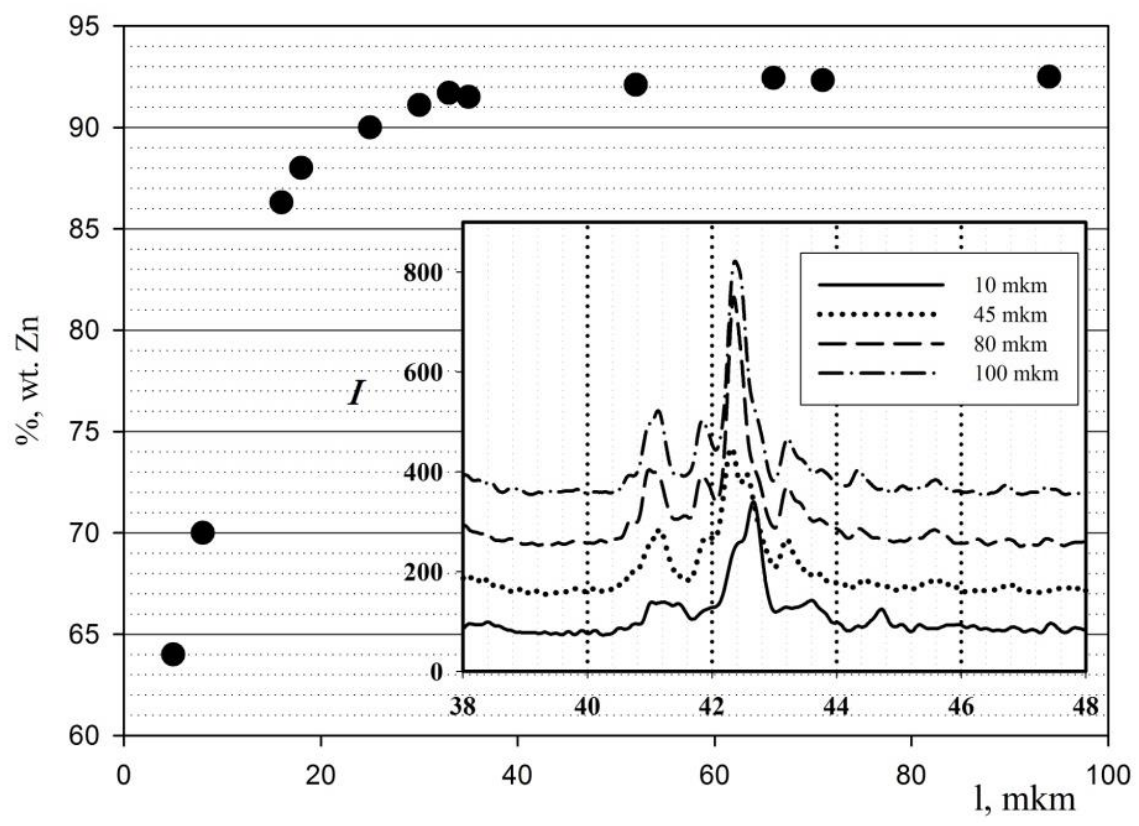

Figure 1. The dependence of the chemical and phase composition on the thickness of DZ coatings (the inset - XRD patterns of DZ coatings).

\subsection{Corrosion and electrochemical behavior}

\subsubsection{Results of gravimetric measurements}

Figure 2 shows the corrosion rate values for coatings with different contents of zinc. These values were obtained gravimetrically.

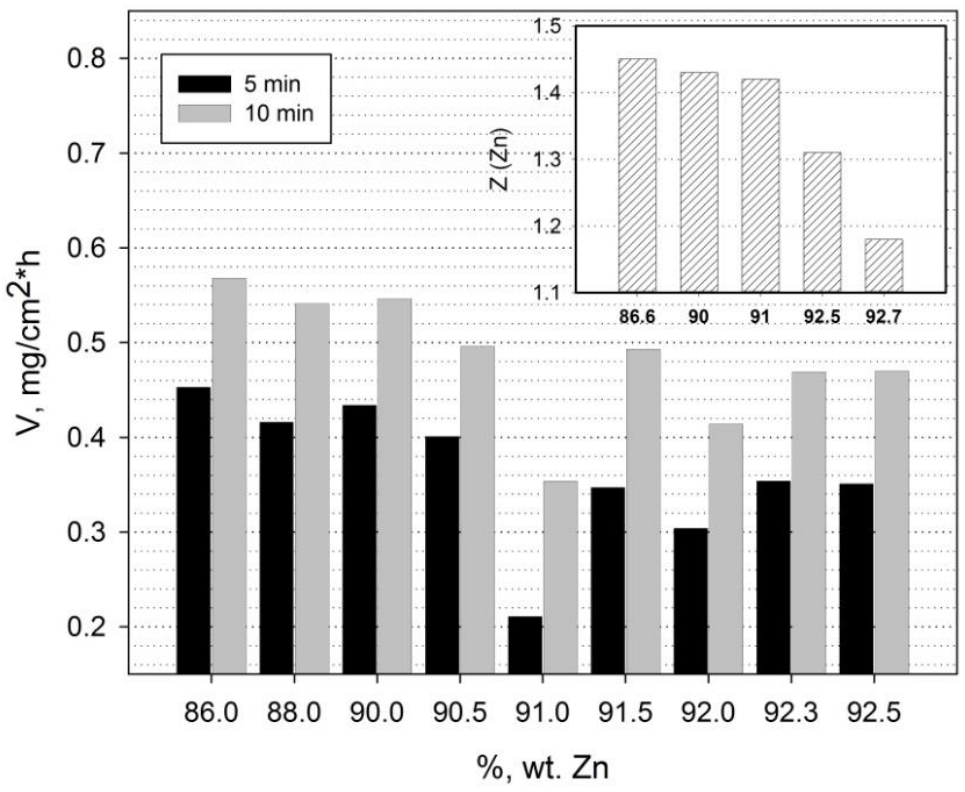

Figure 2. Corrosion rate for DZ coatings with different contents of zinc in a solution of $0.1 \mathrm{M} \mathrm{HCl}+0.5 \mathrm{M} \mathrm{NaCl}$ (exposure 5 and $10 \mathrm{~min}$ ). The inset shows the selectivity coefficients. 
The selectivity coefficient is above 1 and decreases with an increasing concentration of $\mathrm{Zn}$. This indicates that the corrosion of the $\delta$-phase, regardless of its chemical composition, is accompanied by the selective dissolution of zinc. However, with an increasing zinc concentration in the $\delta$-phase, the tendency towards its selective dissolution weakens. This can be explained by a decrease in the concentration of electropositive metal in the alloy and the transition to phases richer in zinc.

The sample of a DZ coating with a zinc concentration of $91.0 \mathrm{wt} . \%$ is characterized by the minimum rate of corrosion, which corresponds to the middle of the $\delta$-phase concentration range. The corrosion rate of the sample is 2 times less than for other coatings. With an increase in zinc content from $86.0 \mathrm{wt} . \%$ to $90.5 \mathrm{wt} . \%$, the corrosion rate shows a slight tendency to decrease. In the concentration range from 91.5 to $92.5 \mathrm{wt} . \%$ of $\mathrm{Zn}$ the corrosion rate takes approximately the same value of $\sim 0.35 \mathrm{mg} / \mathrm{cm}^{2} \cdot \mathrm{h}$. With an increase in the exposure time from 5 to 10 minutes, the corrosion rate increases by $1.2-1.5$ times. The above-described dependence of the corrosion rate on the chemical composition is maintained. However, for the samples of DZ coatings with a zinc concentration of 91.0$91.5 \mathrm{wt} . \%$, the increase in the rate of corrosion is the most significant (by 70\%), while for the remaining coatings it does not exceed $25-35 \%$.

The peculiarities of the corrosion behavior of the coatings can be explained as follows. In case of relatively thin zinc coatings, corrosion is often complicated by the occurrence of a galvanic connection between portions of the coating and the steel substrate, which has a more positive electrode potential. The galvanic connection is possible through the cracks and pores, which appear as a result of the surface development due to the selective dissolution of zinc. An increase in the zinc concentration in DZ coatings is accompanied by an increase in both the total thickness of the coating and the thickness of the $\delta$-phase layer. This reduces the likelihood of galvanic coupling between the surface of the coating and the underlying layers and the steel substrate. Thus, what is observed in the first five minutes of dissolution in the coating of 30-40 $\mu \mathrm{m}$ in thickness with a zinc concentration of $91.0 \mathrm{wt} . \%$ is the corrosion of the $\delta$-phase. Firstly, thinner coatings are more susceptible to the selective zinc dissolution and, secondly, they are prone to galvanic corrosion. An increase in thickness leads to an increase in the influence on the corrosion rate of both corrosion-active zinc and mechanic stresses, which are unavoidable for thick coatings.

\subsubsection{Open circuit potential}

Figure 3 shows the dependence of the open circuit potential of DZ coating on the duration of their exposure to a $0.1 \mathrm{M} \mathrm{HCl}$ solution. 


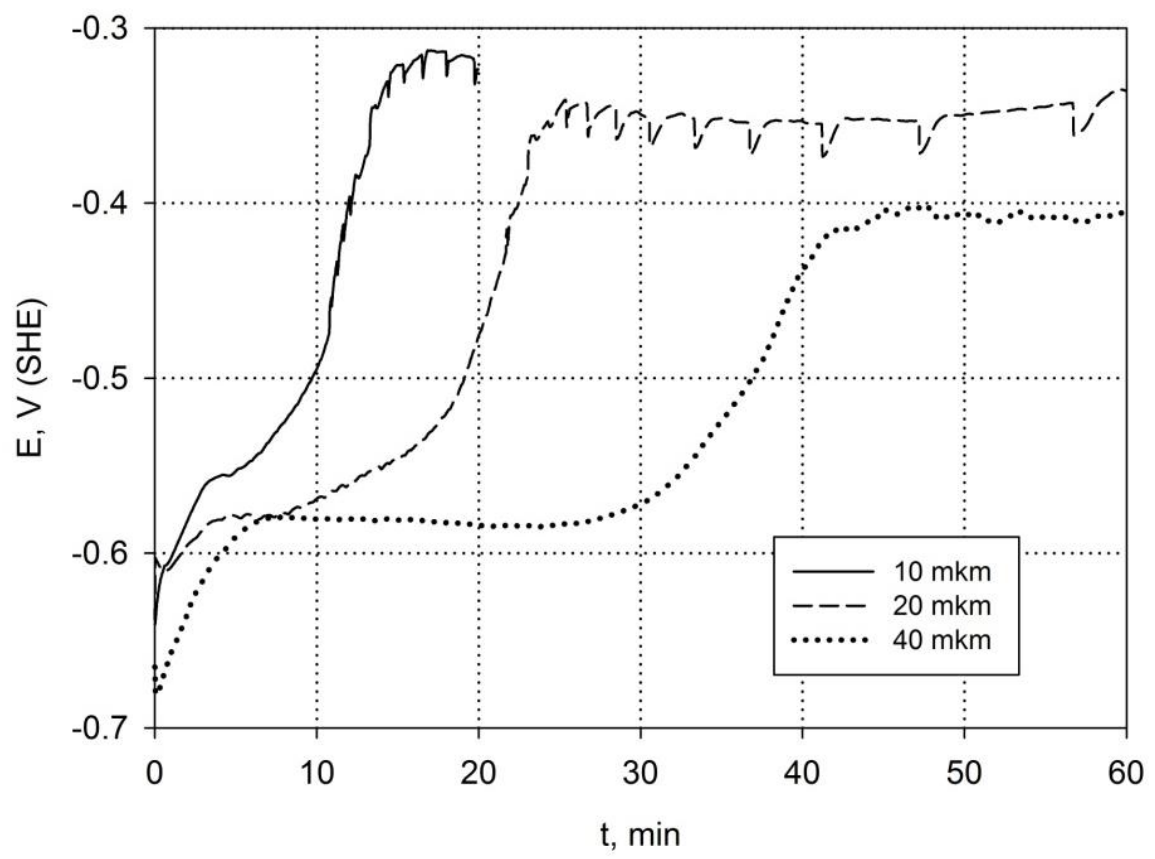

Figure 3. The dependence of the potential coatings on the etching time in a solution of $0.1 \mathrm{M}$ $\mathrm{HCl}$.

Initial potential of a DZ coating with a thickness of $40 \mu \mathrm{m}$, as compared to coatings of 10 and $20 \mu \mathrm{m}$, has a more negative value, which can be explained by a relatively high zinc content in the surface layer. In the first few seconds of exposure, there is a slight reduction in the potential by $20-30 \mathrm{mV}$, which might be associated with the dissolution of the thin oxide film. Then during the next 3-5 minutes, the potential of all three DZ coatings are shifted to the positive side. This is probably due to the predominant dissolution of zinc from the $\delta$-phase. Next, a constant potential $(-0.55 \mathrm{~V}) \ldots(-0.57 \mathrm{~V})$ is established. In the graph, there appears the first plateau, which lasts 3 times less for the coating with a thickness of $20 \mu \mathrm{m}$ than for the coating with a thickness of $40 \mu \mathrm{m}$. The coating with a thickness of $10 \mu \mathrm{m}$ has no expressed plateau and has a slight delay in the increase of potential. This region on the curves can be attributed to the dissolution of the $\delta$-phase.

Further, there is a sharp shift of the potential in the positive direction. What is more, the rate of the $\mathrm{E}$ transformation depends on the thickness: the coating with a thickness of $40 \mu \mathrm{m}$ has the slowest increase in potential. The approach to the second plateau can be seen within the range of $(-0.30 \mathrm{~V}) \ldots(-0.40 \mathrm{~V})$. These values are more negative than the potential of the steel substrate, due to the influence of the $\alpha$-phase. This can be explained by the fact that the layers of iron-zinc phases that constitute a DZ coating are preceded by a solid solution of zinc in iron ( $\alpha$-phase), which is localized in the steel substrate.

It takes more time to obtain coatings of a large thickness, which leads to an increased concentration of zinc in a solid solution and an increase in its thickness. This explains the decrease in potential on the second plateau with an increasing thickness of coatings. During 
this portion, there are potential oscillations that are associated with a release of hydrogen bubbles as a result of corrosion.

\subsubsection{Evolution of coatings' morphology}

When the DZ coatings were dissolved, a transformation of the surface morphology was recorded (Figures 4 and 5). In addition, the chemical composition of the surface and the concentration of zinc and iron ions were determined in the solution.

Figure 4 shows the SEM images of the surface of a DZ coating with a thickness of $20 \mu \mathrm{m}$ after different exposures to a $0.1 \mathrm{M} \mathrm{HCl}$ solution. After 3 minutes of dissolving, there appear cracks and pores of 0.5 to $1.0 \mu \mathrm{m}$ in size (Figure 4a). While it is dissolving, the zinc concentration in the surface layers of the DZ coating decreases by $1.0 \mathrm{wt} . \%$.

As the time of exposure is increased to $10 \mathrm{~min}$, the pores and cracks increase in size (Figure $4 \mathrm{~b}$ ). The concentration of zinc ions at this moment exceeds the concentration of iron in the etching solutions by more than 100 times, and the zinc concentration in the surface layers of the coating is reduced by $10.0 \mathrm{wt} . \%$, which confirms the selective character of dissolution. Uneven dissolution leads to the fact that 5-10 minutes after dissolution a galvanic connection with the steel substrate is formed through the pores and cracks of the coating, which accelerates the corrosion process. On the curve of dependency of the potential on time, this period corresponds to a second sharp increase in potential and its transition to a constant value (the second plateau in Figure 3).

The coating almost completely dissolves after 15 minutes (Figure 4c), which results in the exposed surface of the steel substrate. As a result of further exposure to acid (20 minutes or more), an etching pattern appears, which apparently is a carbon steel microstructure (Figure 4d). The iron content on the surface reaches 95.0-99.0 wt.\%. Zinc is present on the surface in the amount $\approx 0.5 \mathrm{wt} . \%$, which can be explained by the presence of the solid solution of zinc in iron ( $\alpha$-phase) in the upper layers of the steel substrate. After complete dissolution of the coating, the chemical analysis of the etching solutions shows that the ratio of $\mathrm{Fe}: \mathrm{Zn}$ is $1: 10$. This corresponds to the initial ratio of the concentrations of elements in the coating.

Figure $4 \mathrm{e}$ is given for comparison and shows the image of a surface of a coating with a thickness of $40 \mu \mathrm{m}$ after 20 minutes of dissolution. The layer thickness of the $\delta$-phase in this sample is twice as big, therefore, when exposed for 20 minutes, the relatively uniform layer of the $\delta$-phase remains on the surface. This morphology corresponds to the extensive first plateau in the graph showing the dependency of potential over time. SEM images clearly show how the $\delta$-phase morphology changes under the selective dissolution of zinc. Irregular shaped pores with the width of 0.1 to $0.5 \mu \mathrm{m}$ are arranged in random order against $\delta$-phase grains. Over time, the pores increase in size, they multiply and merge into chains, from which cracks are formed leading to the destruction of the coating. 

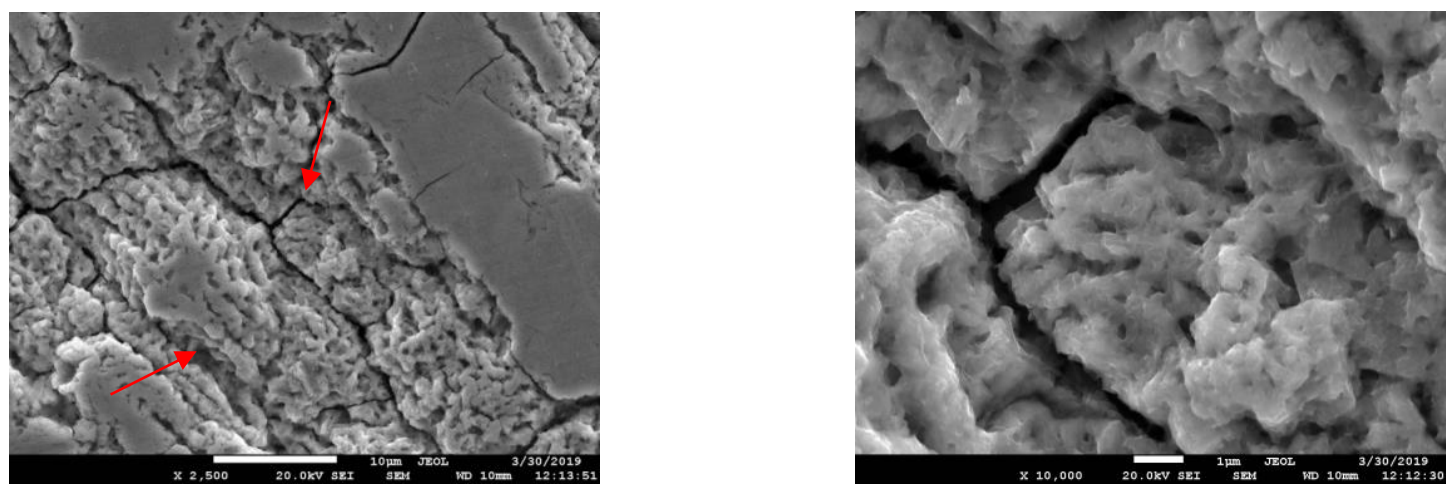

a

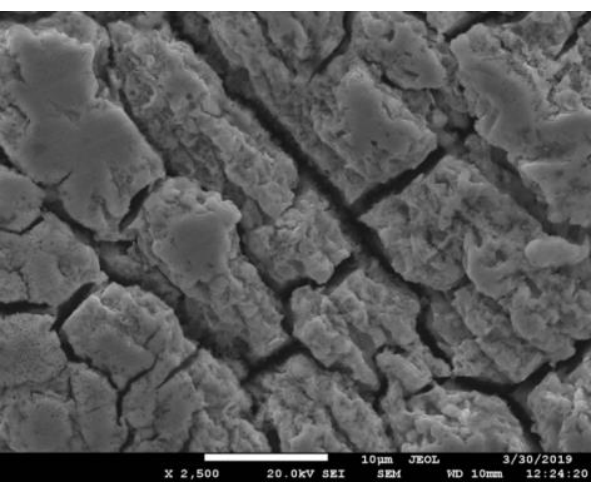

b

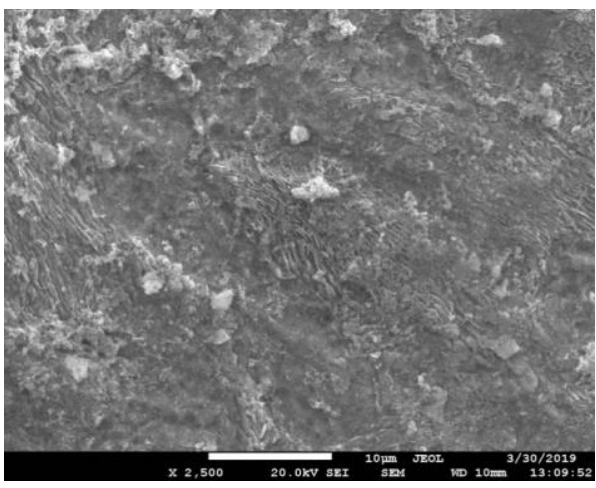

d

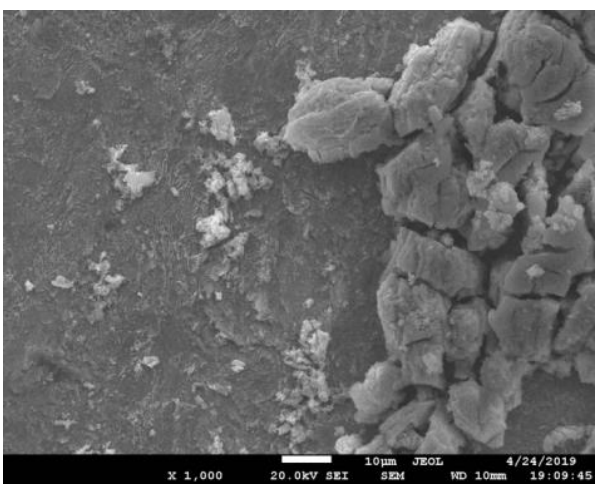

$\mathrm{c}$

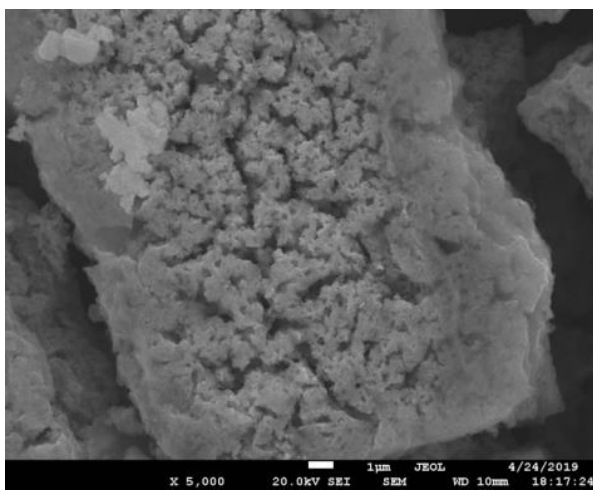

e

Figure 4. SEM images of the surface of DZ coatings of different thicknesses after their etching in a $0.1 \mathrm{M} \mathrm{HCl}$ solution: $\mathrm{a}-\mathrm{d}$, coating of $20 \mu \mathrm{m}$ : $\mathrm{a}-3 \mathrm{~min} ; \mathrm{b}-10 \mathrm{~min} ; \mathrm{c}-15 \mathrm{~min} ; \mathrm{d}-$ $20 \mathrm{~min}$; e -40 coating of $40 \mu \mathrm{m}$, dissolving $20 \mathrm{~min}$.

\subsubsection{Dissolution products}

As a result of prolonged contact of the $\delta$-phase with hydrochloric acid, loose black films appear on the surface of the samples (Figures 5,6). The formation of these films is uneven: first, on separate portions of the surface there appear dark regions which expand and cover 
the entire surface of the sample. This is accompanied by the copious discharge of hydrogen. As a result of prolonged exposure of the sample to acid, the black film gradually exfoliates and is partially dissolved. The time required for the appearance of black areas increases in proportion to the thickness of the coating. Figure 5 shows the SEM images of black structures (shown by arrows).

The formations, which the black film consists of, are of a lighter shade in the SEM images of the surfaces of samples. They are either located near the coating residues (Figure 5a) and directly on the steel substrate or are localized in active centers, such as at the intersections of cracks (Figure 5b). It is quite difficult to determine the chemical and phase composition of the black film directly on the surface as the analysis is affected greatly by the steel substrate.

The film was cleaned off a sample and, as a powder, was subjected to chemical and phase analysis. It was compared with a powder $\delta$-phase, taken from a coating which had not been subjected to dissolution in acid. Figure 6 shows the XRD patterns of the powders and an electron microscope image of a black film powder. According to the results of chemical microanalysis, the concentrations of zinc and iron in the film constitute 67.5 and $32.5 \mathrm{wt} . \%$ respectively. The formations, which the black film consists of, are heterogeneous both in size and the shape of particles. The surface of particles is covered with tiny pores, which apparently makes the film very active in terms of corrosion. The determined zinc concentration is $4.5 \%$ less than the lower concentration limit for the gamma-phase $\left(\mathrm{Fe}_{3} \mathrm{Zn}_{10}\right)$ and according to the Fe-Zn phase diagram, such concentrations of iron and zinc correspond to the coexistence of the gamma-phase and a solid solution of $\mathrm{Zn}$ in $\mathrm{Fe}$ [1]. However, in the XRD patterns of the black film within an angle range of $40-442 \theta$, there are peaks characteristic of the $\delta$-phase $\left(\mathrm{FeZn}_{7-10}\right)$, wherein the concentration of iron should not exceed $10-12$ wt. $\%$.

It can be assumed that the black film is a $\delta$-phase, coated with products of the $\delta$-phase dezincification accompanied by the formation of fine X-ray amorphous iron. The black color of the resulting film is apparently linked to the small size of the particles of the released iron and the pores between them, which also explains the absence of iron reflections on the XRD pattern of the film.

\section{Conclusions}

In the acidic and weak acidic media, the $\delta$-phase of diffusion zinc coatings is predominately subjected to a selective dissolution of zinc. The rate of the dissolution non-linearly depends on the zinc concentration and the thickness of the coating. The $\delta$-phase dezincification is accompanied by the morphological development of the surface and the formation of multiple cracks and pores, which, in their turn, initiate a strong galvanic corrosion. As a result of prolonged corrosion in an acidic medium, the iron-zinc coating is subjected to a deep dezincification with a phase transformation. 


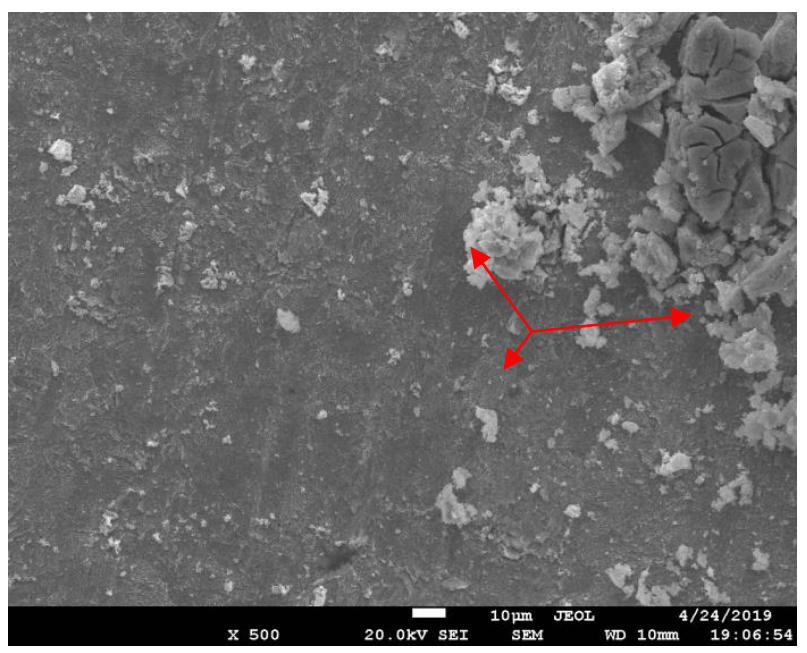

a

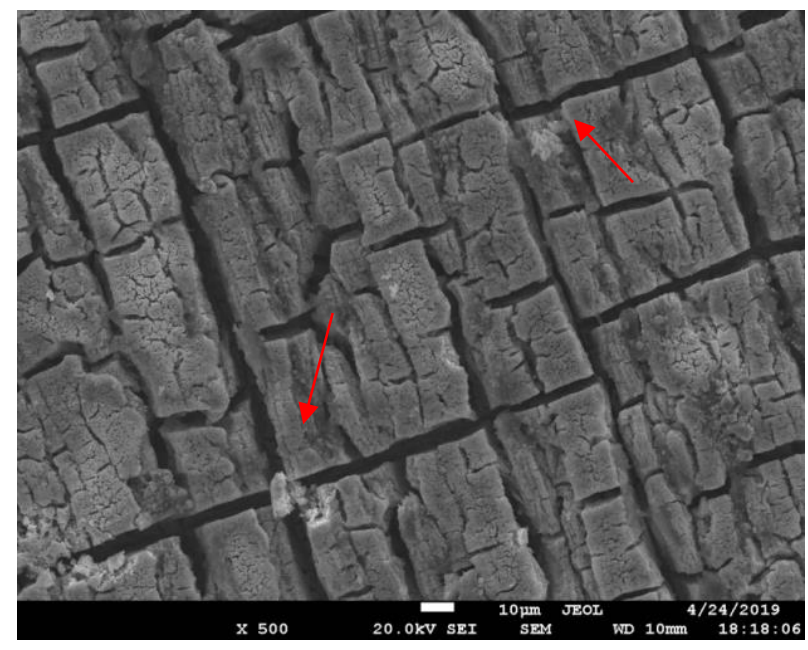

b

Figure 5. SEM images of the surface of DZ coatings after their etching in a $0.1 \mathrm{M} \mathrm{HCl}$ solution: $\mathrm{a}$ - thickness of $20 \mu \mathrm{m}, 15 \mathrm{~min}$; $\mathrm{b}$ - thickness of $40 \mu \mathrm{m}, 20 \mathrm{~min}$.

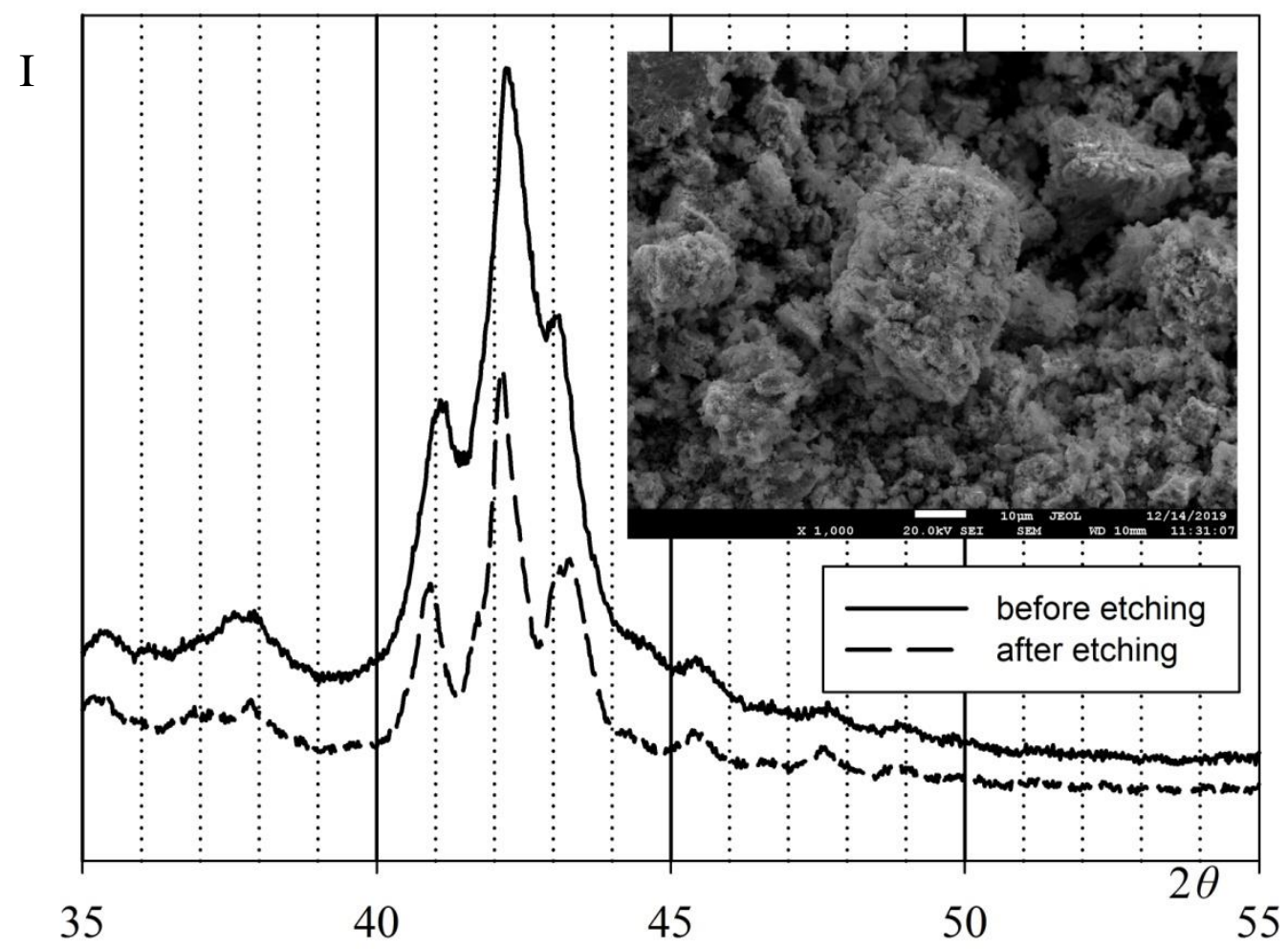

Figure 6. XRD patterns of the powder $\delta$-phase before and after dissolution in acid (the inset SEM image of a black film). 


\section{References}

1. A.R. Marder, The metallurgy of zinc-coated steel, Prog. Mater. Sci., 2000, 45, no. 3, 191-271. doi: 10.1016/S0079-6425(98)00006-1

2. E.V. Proskurkin and N.S. Gorbunov, Galvanizing, sherardizing and other zinc diffusion coatings, Zinc Development Assoc., London and Technicopy Ltd., Gloucestershire, GL 102 NA, England, 1975, 261.

3. F. Natrup and W. Graf, Sherardizing: corrosion protection of steels by zinc diffusion coatings, Thermochemical Surface Engineering of Steels, Woodhead Publishing, England, 2015, 737-750. doi: 10.1016/C2013-0-16318-0

4. X.G. Zhang, Corrosion and electrochemistry of zinc, Springer Science \& Business Media, USA, 2013, 463.

5. I. Odnevall Wallinder and C. Leygraf, A critical review on corrosion and runoff from zinc and zinc-based alloys in atmospheric environments, Corrosion, 2017, 73, no. 9, 1060-1077. doi: $\underline{10.5006 / 2458}$

6. W.J. Ooij and A. Sabata, Under-vehicle corrosion testing of primed zinc and zinc alloycoated steels, Corrosion, 1990, 46, no. 2, 162-171. doi: $10.5006 / 1.3585083$

7. E. Almeida and M. Morcillo, Lap-joint corrosion of automotive coated materials in chloride media. Part 3 - Electrogalvanized steel/galvanneal interface, Surf. Coat. Technol., 2000, 124, no. 1, 44-52. doi: 10.1016/S0257-8972(99)00625-8

8. R.G. Galin, A.I. Biryukov, D.A. Zakharyevich, T.V. Batmanova, E. Yankov and S. Fisckuchev, Corrosion properties of diffusional zinc coatings obtained by nanogalvanizing, Mater. Sci. Nonequilib. Phase Transform., 2017, 3, no. 4, 165-166.

9. A.I. Biryukov, R.G. Galin, D.A. Zakharyevich, A.V. Wassilkowska and T.V. Batmanova, The effect of the chemical composition of intermetallic phases on the corrosion of thermal diffusion zinc coatings, Surf. Coat. Technol., 2019, 372, 166-172. doi: 10.1016/j.surfcoat.2019.05.029

10. A.I. Biryukov, R.G. Galin, D.A. Zakharyevich, A.V. Wassilkowska, A.V. Kolesnikov and T.V. Batmanova, A layer-by-layer analysis of the corrosion properties of diffusion zinc coatings, Arch. Metall. Mater., 2020, 65, no. 1, 99-102. doi: 10.24425/amm.2019.131101

11. O.A. Kozaderov, O.V. Koroleva and A.V. Vvedenskii, Kinetics of phase transformations in a binary alloy surface layer at the selective dissolution. I. Theoretical analysis, Prot. Met. Phys. Chem. Surf., 2009, 45, no. 1, 31-35. doi: 10.1134/S2070205109010043

12. A.V. Vvedenskii, S. Grushevskaya, D. Kudryashov and S. Ganzha, The influence of the conditions of the anodic formation and the thickness of $\mathrm{Ag}(\mathrm{I})$ oxide nanofilm on its semiconductor properties, J. Solid State Electrochem., 2010, 14, 1401-1413. doi: $\underline{10.1007 / \mathrm{s} 10008-009-0952-9}$ 
13. O.A. Kozaderov and A.V. Vvedenskii, Voltamperometry of selective dissolution of Ag$\mathrm{Au}$ alloys under conditions of solid phase-liquid phase mass transfer, Prot. Met. Phys. Chem. Surf., 2013, 49, no. 6, 724-733. doi: 10.1134/S2070205113060099

14. P. Volovitch, T.N. Vu, C. Allély, A.A. Aal and K. Ogle, Understanding corrosion via corrosion product characterization: II. Role of alloying elements in improving the corrosion resistance of $\mathrm{Zn}-\mathrm{Al}-\mathrm{Mg}$ coatings on steel, Corros. Sci., 2011, 53, no. 8, 2437-2445. doi: $10.1016 / j$.corsci.2011.03.016

15. X.G. Zhang and I.C. Bravo, Electrochemical stripping of galvannealed coatings on steel, Corrosion, 1994, 50, no. 4, 308-317. doi: $10.5006 / 1.3294338$

16. F.M. Queiroz and I. Costa, Electrochemical, chemical and morphological characterization of galvannealed steel coating, Surf. Coat. Technol., 2007, 201, no. 1617, 7024-7035. doi: 10.1016/j.surfcoat.2007.01.005

17. H.Y. Ha, S.J. Park, J.Y. Kang, H.D. Kim and M.B. Moon, Interpretation of the corrosion process of a galvannealed coating layer on dual-phase steel, Corr. Sci., 2011, 53, no. 7, 2430-2436. doi: 10.1016/j.corsci.2011.04.001

18. T. Bellezze, S. Saltykov, G. Roventi, M. Malavolta and R. Fratesi, Layer-by-layer analysis of hot-dip zinc coating by anodic dissolution in acetate bath, Surf. Coat. Technol., 2012, 206, no. 24, 5023-5027. doi: 10.1016/j.surfcoat.2012.04.008

19. C. Xhoffer, H. Dillen, B.C. De Cooman and A. Hubin, Quantitative phase analysis of galvannealed coatings by coulometric stripping, J. Appl. Electrochem., 1999, 29, no. 2, 209-219. doi: $10.1023 / \mathrm{A}: 1003435824284$

20. A.P. Yadav, H. Katayama, K. Noda, H. Masuda, A. Nishikata and T. Tsuru, Effect of $\mathrm{Fe}-\mathrm{Zn}$ alloy layer on the corrosion resistance of galvanized steel in chloride containing environments, Corr. Sci., 2007, 49, no. 9., 3716-3731. doi: 10.1016/j.corsci.2007.03.039

21. R. Mishra, Quantification of galvannealed coating phases using the galvanostatic technique, Metall. Mater. Trans. A, 2008, 39, no. 10, 2275-2277. doi: $10.1007 / \mathrm{s} 11661$ $\underline{008-9592-4}$

22. R.M.V. Paranhos, V.F.C. Lins, A.A.M. Waldemar and E.A. Alvarenga, Optimization of electrochemical stripping of galvannealed interstitial free steels, Surf. Eng., 2011, 27, no. 9, 676-682. doi: 10.1179/1743294410Y.0000000015

23. H. Kaiser, De-alloying and dissolution induced cracking of the zinc-iron $\delta$ phase, Mater. Corros., 1996, 47, no. 1, 34-41. doi: 10.1002/maco.19960470106

24. S.M. Jiang, D.W. Guo, Q.F. Zhang, The structure and corrosion process characterization of galvannealed and galvanized steel sheets, Adv. Mater. Res., 2014, 834, 589-596. doi: 10.4028/www.scientific.net/AMR.834-836.589

25. R.G. Galin, Modified zinc powder, Russian Patent, 2170643, C23C10/28, MPK B22F1/02, 2001.

26. R.G. Galin, D.A. Zakharyevich and S.V. Rushchits, Formation and structure of diffusional zinc coatings formed in nanocrystallized zinc powders, Mater. Sci. Forum, 2016, 870, 404-408. doi: 10.4028/www.scientific.net/MSF.870.404 